\title{
Controlling waves in space and time for imaging and focusing in complex media
}

\begin{abstract}
Allard P. Mosk ${ }^{1 \star}$, Ad Lagendijk ${ }^{1,2}$, Geoffroy Lerosey ${ }^{3}$ and Mathias Fink ${ }^{3}$
In complex media such as white paint and biological tissue, light encounters nanoscale refractive-index inhomogeneities that cause multiple scattering. Such scattering is usually seen as an impediment to focusing and imaging. However, scientists have recently used strongly scattering materials to focus, shape and compress waves by controlling the many degrees of freedom in the incident waves. This was first demonstrated in the acoustic and microwave domains using time reversal, and is now being performed in the optical realm using spatial light modulators to address the many thousands of spatial degrees of freedom of light. This approach is being used to investigate phenomena such as optical super-resolution and the time reversal of light, thus opening many new avenues for imaging and focusing in turbid media.
\end{abstract}

$\mathrm{V}$ isible light is our prime means of collecting information about the microscopic and macroscopic structure of matter. Unfortunately, refractive-index inhomogeneities cause light to be strongly scattered in many materials of technological and biomedical relevance ${ }^{1-3}$. The scattering strength is expressed in terms of the scattering mean free path $l$, which is the average distance waves propagate between scattering events. When a beam of light enters a thin scattering medium, one can observe a beam of refracted ('ballistic') light that is exponentially extinguished as it progresses deeper into the medium. In a non-absorbing medium, the energy in the beam is not lost, but is instead converted into a diffuse glow of scattered light. This light hides the internal structure of any material thicker than a few multiples of $l$, and thus represents a major obstacle to the imaging and focusing of light in fields such as biomedical imaging ${ }^{4}$, laser therapy ${ }^{5}$, art preservation ${ }^{6}$ and photonic crystal fabrication ${ }^{7}$.

Early experiments in holography ${ }^{8}$ showed that light scattering by time-independent media does not lead to an irretrievable loss of information ${ }^{9}$. Instead, the information is scrambled into disordered interference patterns called laser speckles ${ }^{10}$. The combination of statistical optics and mesoscopic transport theory developed for electrons ${ }^{11,12}$ led to proposals for extracting imaging information from laser speckles ${ }^{9,13}$. The ability to manipulate interference in multiply scattered light has recently given rise to new focusing and imaging techniques in which scattering enables - rather than impedes the focusing of light. This Review covers the rapid progress made in recent years towards the goal of controlling of waves in strongly scattering media.

There are many innovative techniques for imaging inside scattering media. One successful approach is to reject, by a variety of elegant methods, waves that have been multiply scattered. Optical coherence tomography ${ }^{14}$ rejects the scattered light by selecting the signal based on its propagation time. In multiphoton microscopy ${ }^{15}$, scattered light is rejected because its intensity is too low to contribute to nonlinear optical processes. However, at depths larger than $5 l$, more than $99 \%$ of the light has undergone multiple scattering and methods that reject scattered light therefore cannot be used. The diffuse glow of multiply scattered light can be used to locate objects $^{16}$ and provide optical tomography at very large depths ${ }^{4}$. The low resolution of such tomographic techniques can be improved by combining ultrasound and optical methods ${ }^{17}$. In media such as the atmosphere, which scatter light much more weakly than the systems we consider here, many beautiful methods ranging from adaptive optics $^{18,19}$ to stellar speckle interferometry ${ }^{20}$ allow for the distortionfree imaging of distant objects.

\section{Wave propagation in scattering media}

Light propagation in complex media is often described as the diffusion of particles that perform a random walk. Although the particle-diffusion approach and radiative transfer theory ${ }^{21}$ neglect the interference terms responsible for enhanced backscattering ${ }^{22,23}$ and strong localization ${ }^{24-27}$, they are both remarkably effective at predicting the average intensity distribution ${ }^{28}$. The diffusion approach is concerned with the ensemble-averaged intensity, which is always smooth. In contrast, when light is scattered on a single disordered sample, a grainy intensity pattern - the laser speckle - is observed.

The propagation of light is described by a wave equation that relates the evolution in time and space of a wave field $\Psi$, such that $\nabla^{2} \Psi(\mathbf{r}, t)=n^{2}(\mathbf{r}) c^{-2} \partial^{2} \Psi(\mathbf{r}, t) / \partial t^{2}$. Here, $\Psi(\mathbf{r}, t)$ can represent the electric field of light (suppressing, for simplicity, the polarization degree of freedom), the pressure of longitudinal sound or a Schrödinger probability amplitude. The propagation speed of the waves is denoted by $c / n(\mathbf{r})$, where $c$ is the speed of light in vacuum and $n(\mathbf{r})$ is the index of refraction. Scattering is caused by local variations of $n(\mathbf{r})$, which can occur, for example, due to the presence of small dielectric particles. In clear media, $n(\mathbf{r})$ is constant and the wave equation can be solved to obtain the wave's normal modes, such as plane waves or linear combinations of plane waves. Only a finite number of independent transversal modes carry energy from the surface area of the system to the far field and vice versa - these are the incident and outgoing transversal modes of the system. A system with surface area $A$ allows $N_{\mathrm{S}}=2 \pi A / \lambda^{2}$ independent incident modes for light of wavelength $\lambda$. Visible light has around 10 million transversal modes per square millimetre. Any incident wave from the far field can be decomposed into these incident modes, which therefore represent the spatial degrees of freedom of the incident light field. These transversal modes define the basis vectors of a transmission matrix whose many millions of elements fully describe wave transmission through the sample $e^{11,12}$. 


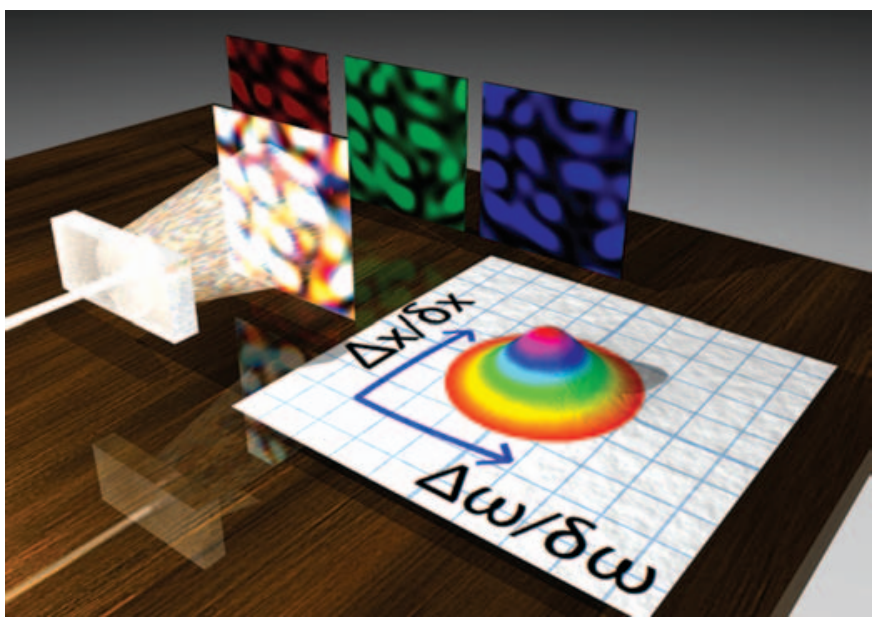

Figure 1 | Speckle correlations in space and frequency. When a whitelight beam is incident on a multiply scattering medium, frequency components that are spaced more than the correlation frequency $\delta \omega$ give rise to uncorrelated speckle patterns. The blue and red patterns symbolize frequency components spaced by $\delta \omega$; the green pattern is intermediate. Spatial correlations are lost when the beam is moved by more than one correlation width (the 'speckle size'). The speckle correlation graph shows how speckle correlations are lost as the beam is moved in space or frequency.

The microscopic wave equation exhibits time-reversal symmetry: if $\psi(\mathbf{r}, t)$ is a solution, so is its time-reversed equivalent $\psi(\mathbf{r},-t)$. An outward-propagating wave whose intensity and phase pattern resemble a random speckle can be time-reversed to reach a focus at the point of origin. Scattering from a stationary disorder does not break this time reversal symmetry; the time-reversed waves undergo time-reversed scattering to form a high-intensity focus ${ }^{29}$.

The ability to focus waves by interference is intimately connected to the spatial and temporal speckle correlation functions. In an open, non-absorbing medium, energy enters and leaves the system at the edges. The typical time a photon spends traversing a medium of thickness $L$ is the Thouless time, $\tau_{\mathrm{D}} \approx L^{2} /\left(l_{\mathrm{e}}\right)$, where $v_{\mathrm{e}}$ is the energy velocity ${ }^{30-32}$. In an open medium, the solutions of the wave equation can be expanded into quasimodes with a frequency width of the order of $\delta \omega=1 / \tau_{\mathrm{D}}$ (refs 2,33 ). Two incident light waves with a frequency spacing smaller than $\delta \omega$ will produce strongly correlated transmitted fields, whereas waves with a frequency difference larger than $\delta \omega$ couple to completely different solutions of the wave equation. The correlations of multiply scattered light in frequency and space are illustrated in Fig. 1. Speckle correlations at the surface of the sample decay with a typical width of $\delta x \approx \lambda / 2 n(\mathbf{r})$, which corresponds to the average size of a speckle ${ }^{34,35}$. Because transmission through the sample depends on frequency differences of $\delta \omega$ or more, frequency components separated by $\delta \omega$ are considered to be different degrees of freedom of the incident field. A broadband field, which has a bandwidth of $\Delta \omega \gg \delta \omega$, couples to a very large number $N$ of incident spatiotemporal degrees of freedom, giving $N=N_{\mathrm{S}} N_{\mathrm{f}}=N_{\mathrm{S}} \Delta \omega / \delta \omega$, where $N_{\mathrm{S}}$ is the number of spatial modes and $N_{\mathrm{f}}$ is the number of independent frequencies ${ }^{36}$. In the diffusive regime, which is typical for most optical experiments, a large fraction of these incident modes is transmitted with reasonable efficiency, and control over the incident degrees of freedom provides control over the transmitted and internal fields. In regimes where waves are confined by waveguides or photonic crystals, the number of available transmitted modes may be small, and control over the transmitted field is incomplete ${ }^{37}$. In such cases, control of many incident degrees of freedom is still important because it allows the light to be coupled efficiently to the few available transmission channels.
Most theoretical descriptions of wave propagation are appropriate for any kind of wave, be it electromagnetic, acoustic or even a matter wave. However, a large difference exists in the hardware available at particular frequencies: in ultrasound and radiofrequency experiments it is typically possible to record and synthesize waveforms over the full signal bandwidth. Although this gives access to thousands of frequency degrees of freedom, the cost and size of the transducers required mean that only a small number (typically up to 100) spatial channels can be addressed. In the optical domain, however, CCD cameras and spatial light modulators (SLMs) ${ }^{38}$ are able to address millions of spatial degrees of freedom, but within only a narrow frequency bandwidth.

\section{Using spatial degrees of freedom to control light}

The first demonstrations of using interference to control multiply scattered light were made by Vellekoop and co-workers ${ }^{39}$, initially by focusing light transmitted through a layer of strongly scattering material and soon after by focusing light onto a fluorescent probe deep inside a strongly scattering layer ${ }^{40}$. They optimized thousands of degrees of freedom of the incident wavefront using the measured intensity at one - or several - targets as a feedback signal. Each incident mode gives rise to a different speckled field behind the sample (Fig. 2a). Feedback from the target forms a linear combination of incident modes that constructively interferes at the target point (Fig. 2a, right). An unoptimized plane wave that is incident on the sample gives rise to a speckle transmission pattern (Fig. 2b, top) and an SLM is then used to optimize the phase of thousands of incident modes. The phase of each mode is modulated through $2 \pi$ and the phase that leads to optimal intensity in the target is kept. This method requires only a detector at the target location, and the optimization scheme is similar to phased array methods in adaptive optics ${ }^{18}$, which compensate for phase distortions in clear air caused by turbulence. After cycling the $N$ controlled incident modes, the intensity on the target is enhanced relative to the uncontrolled initial speckle by a factor of $\eta=N \pi / 4$. Experimental intensity enhancements of up to $\eta=1,080$ have been reported (Fig. $2 b$, bottom) ${ }^{39}$. For higher degrees of freedom, sample drift and measurement noise limited the achievable enhancement in the initial work of Vellekoop and co-workers. Defocusing or aberration in the SLM had no discernible effect on the optimization results.

The statistical properties of wave propagation in scattering media can be understood by employing mesoscopic transport theory ${ }^{12}$. An important concept in this theoretical framework is the notion of open and closed transport channels. Such channels are linear combinations of the incident free propagation modes that are specific to the sample configuration. Fully open channels are transmitted diffusely with almost no back reflection, whereas closed channels have exponentially small transmission. Dorokhov ${ }^{41,42}$ showed that the transmission of waves through a scattering sample is due to a small number of open transmission channels. Fluctuations in the coupling between the free propagation modes and the open transmission channels were shown by Imry to be the cause of conductance fluctuations in electronics ${ }^{43}$.

A wavefront-shaping experiment provided the first direct observation of open transmission channels ${ }^{44}$. In this work, researchers controlled and optimized a large fraction (around 50\%) of all incident modes to form a focus with an intensity that was 700 times stronger than the background. Under these conditions, evidence that light coupled into the open channels was provided by a $40 \%$ increase in transmission outside the bright spot. This increased background transmission led to a lower contrast between the focus and the optimized background. Numerical calculations presented an image of the open channels ${ }^{45}$ that supported the experimental findings. In applications that require power to be transmitted through a scattering medium, one may inject light into open channels to make use of their higher transmission. Indeed, in the microwave regime, 

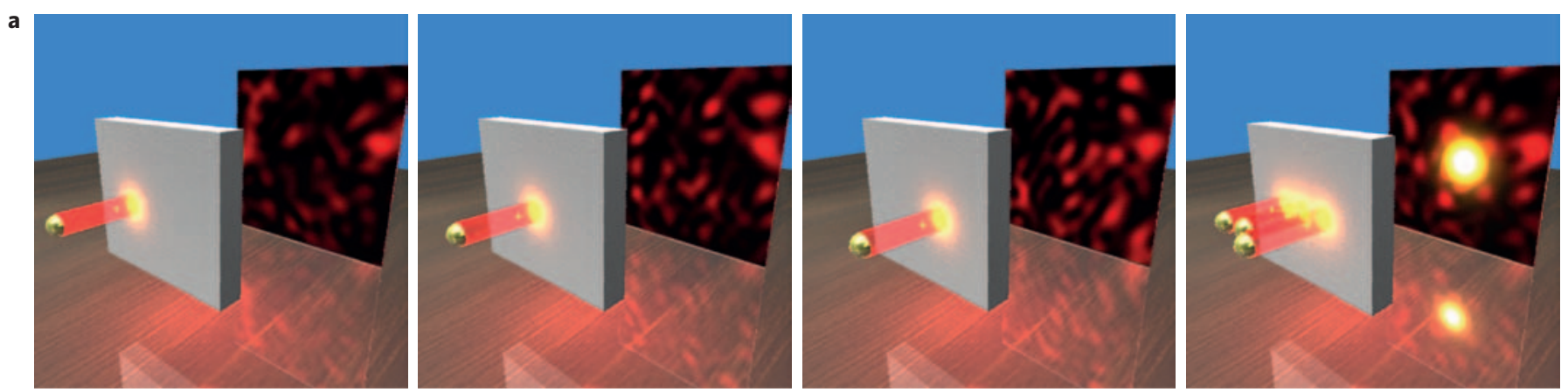

b
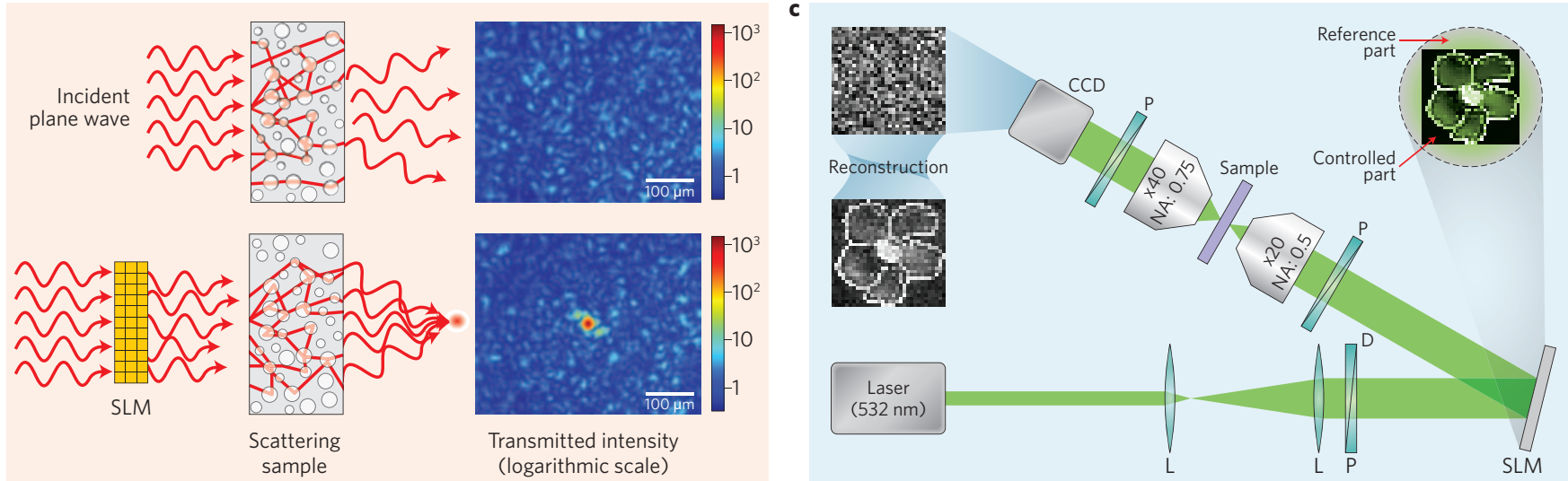

Figure 2 | Using spatial degrees of freedom to focus light through complex media. a, Monochromatic light beams incident at different positions on a multiply scattering medium give rise to uncorrelated transmitted field patterns. A shaped wavefront (right) can be seen as a properly phased combination of a large number of incident beams (only three shown), which gives rise to a bright transmitted focus. $\mathbf{b}$, Schematic of a wavefront-shaping experiment. Thousands of incident modes of the light field are phase-controlled by an SLM. When the phase is optimized, the intensity at the target is over 1,000 times the initial speckle intensity. c, A strongly scattering medium - whose transmission matrix has been measured - can act as a lens. The transmission matrix is found by measuring the sample's response to a large number of incident fields. The transmitted light is imaged by a CCD camera. The phase is determined with the help of a scattered reference field, for which a part of the SLM is reserved. The image of an object projected onto the sample can be reconstructed from the transmitted speckle field. L, lens; P, polarizer; D, diaphragm. Figure b adapted from ref. 39, (c) 2007 OSA. Figure c reproduced from ref. 50, (c) 2010 NPG.

researchers have identified open channels with transmission close to unity ${ }^{46}$. The excitation of such channels, which is an experimental challenge yet to be accomplished, could lead to a tenfold increase in the transmission of a disordered waveguide.

Optimization of an incident wavefront enables several transmitted modes to be controlled simultaneously. The optimization procedure is equivalent to measuring a single row of the medium's transmission matrix. A direct measurement of the full matrix is desirable in order to completely control light transmission and thus investigate all the statistical properties of the transmission matrix. Popoff and co-workers demonstrated the first optical measurement of over 60,000 elements of a disordered sample's transmission matrix ${ }^{47}$. Their measurement set-up is shown in Fig. 2c. Light from a laser is spatially modulated by an SLM and then transmitted through a sample, as in a traditional wavefront-shaping set-up. However, instead of detecting the intensity at a single target point, a camera detects a large number of transmitted field modes. The phase of the transmitted modes is recovered relative to a scattered reference, which is just a small portion of the transmitted light. Although 60,000 was (at the time) an unprecedented number of matrix elements, it represented only a small fraction of the full matrix, which comprised millions of elements. Having the complete transmission matrix, or even a fraction of it, allows the light transmitted through the sample to be focused at a desired location without the need for further optimization. Moreover, it also allows the statistical properties of the transmission matrix to be examined directly ${ }^{48}$. Knowledge of the transmission matrix allows a scattering sample to be used as a high-quality optical element, as first suggested by Freund ${ }^{9}$. Kohlgraf-Owens and Dogariu demonstrated that a scattering medium with a known transmission matrix can be used as either a polarimeter or a spectrometer ${ }^{49}$. Popoff and co-workers demonstrated the ability to image using a scattering medium with a known transmission matrix as a lens ${ }^{50}$. They projected an image on their sample and made a phase-sensitive measurement of the transmitted light, which appeared totally uncorrelated to the original image. However, using the information in the transmission matrix, the researchers were able to reconstruct the image (Fig. 2c).

The wavefront-shaping procedure used by Vellekoop and coworkers ${ }^{39,40}$ is mathematically equivalent to phase conjugation ${ }^{44}$. In 1966, Leith and Upatnieks gave a dramatic demonstration of how phase conjugation by holographic methods can counteract phase scrambling due to single scattering ${ }^{8}$. Optical phase conjugation has been achieved through holography, nonlinear optics ${ }^{51}$, Brillouin scattering $^{52}$ and the use of photorefractive crystals ${ }^{53}$. In 2008, Yaqoob and co-workers provided an implementation of phase conjugation for reversing wavefronts in biological tissue ${ }^{54}$. Using a weak phase-conjugate beam from a photorefractive crystal, the researchers were able to transmit a hologram through a slice of chicken tissue. Although this was a powerful demonstration of the imagetransmission capabilities of scattering media, the image remained on the same side of the medium as the original. Combining wavefront measurement with the use of SLMs makes it possible to create phase-conjugate waves with great flexibility ${ }^{55}$, which is expected to become a key technique in imaging applications. 

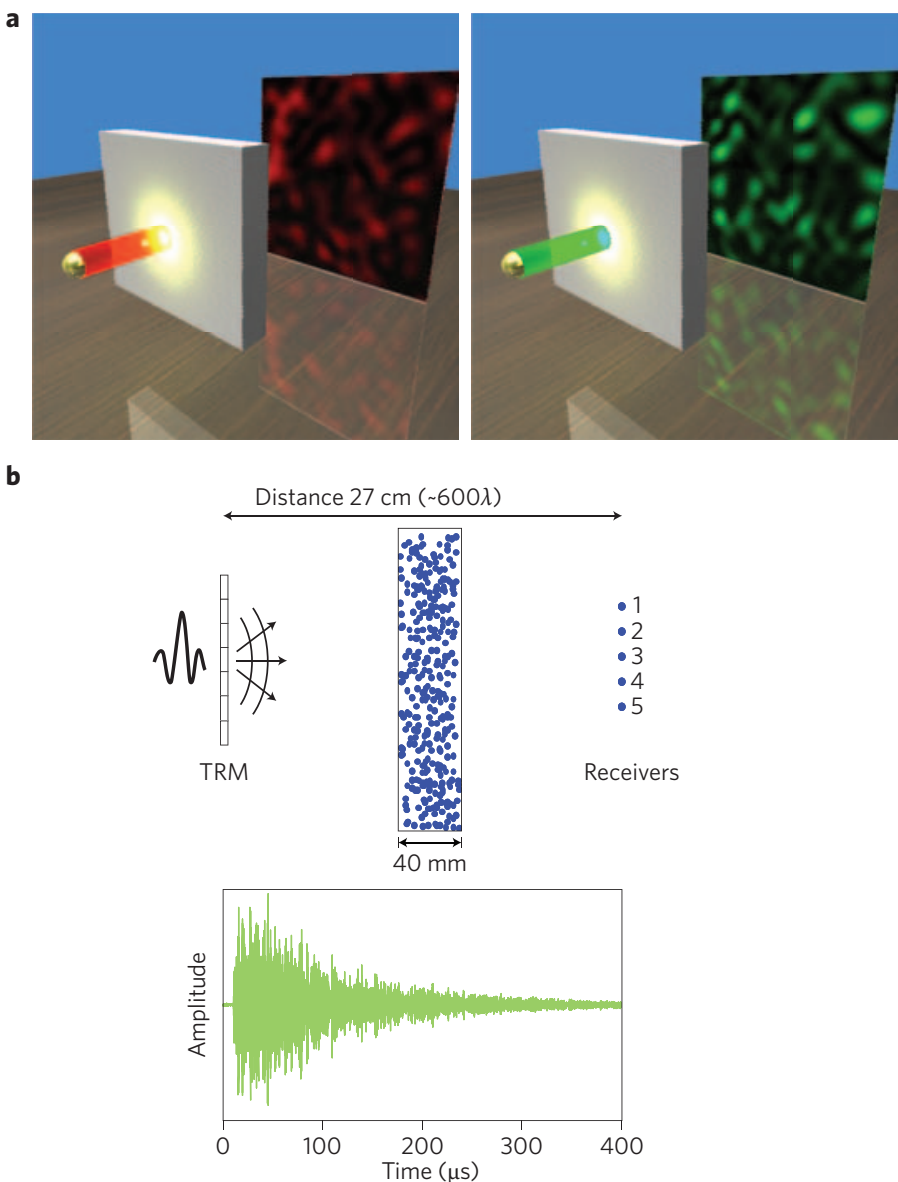

With scattering medium

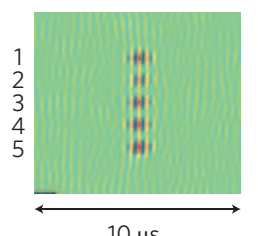

$10 \mu \mathrm{s}$

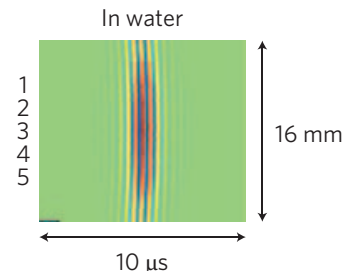

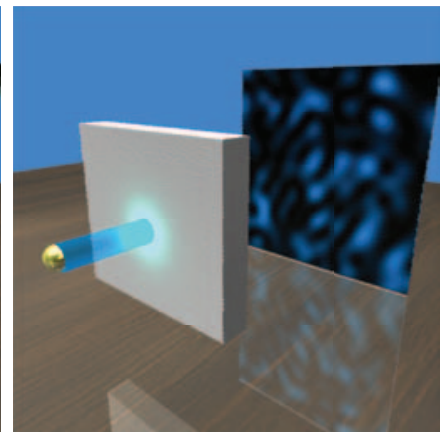

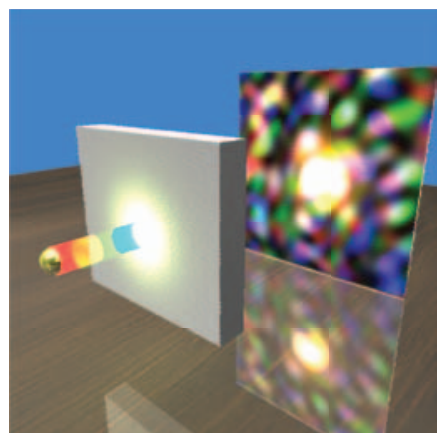

c

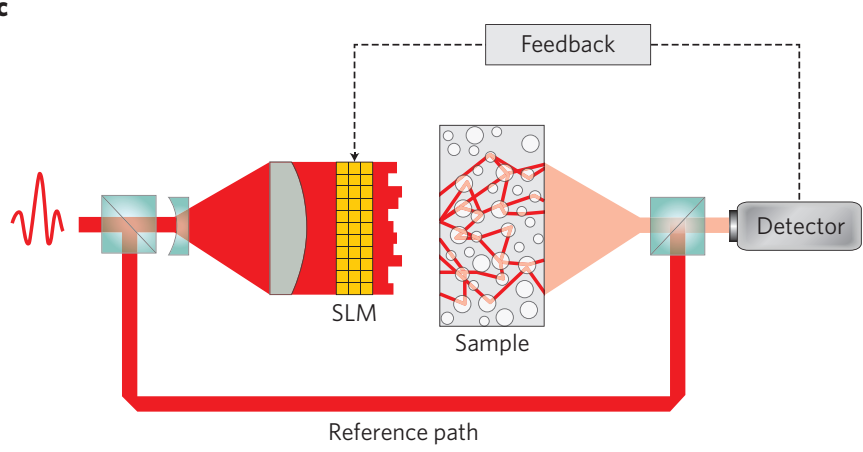

Reference path

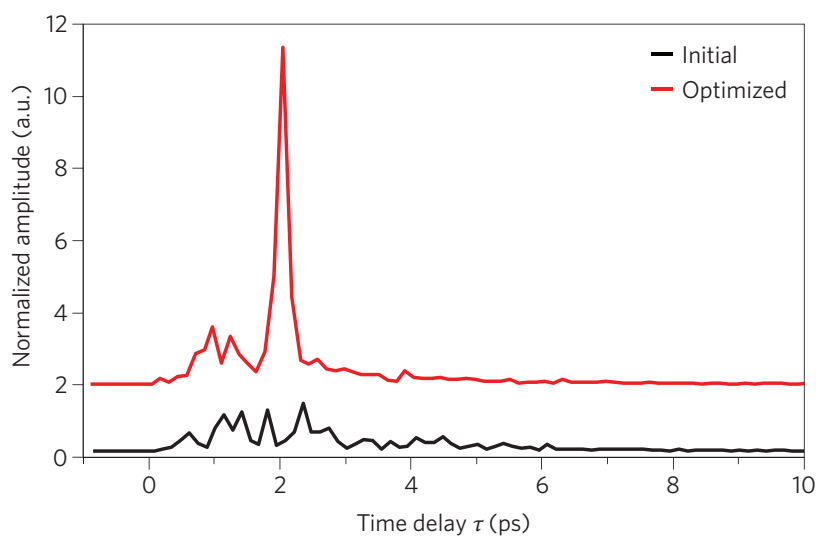

Figure 3 | Taking advantage of the temporal degrees of freedom in complex media. a, A red laser beam creates a speckle pattern after propagating through a multiply scattering medium. Shifting the laser frequency by more than the correlation frequency of the medium provides an uncorrelated speckle pattern, which is depicted here by the green and blue speckles. A focus in space and time can be created by adjusting the phases and amplitudes of the frequency components. b. Top: Selective focusing of ultrasound waves using time reversal. A 1- $\mu$ s-duration pulse centred at $1.5 \mathrm{MHz}$ is emitted from each transducer of a TRM and propagates through a multiply scattering medium towards five receivers. Middle: Example of a 400- $\mu$-long impulse response acquired from a TRM/receiver couple. Bottom: Time reversal is used to focus waves spatiotemporally onto five independent foci through the scattering medium; a single spot is measured through water. c, Optical pulse transmission through a disordered medium. A 65 fs pulse from a Ti:sapphire laser is passed through an SLM before it hits a scattering sample. The scattered pulse has a duration of several picoseconds. Using a reference path, the transmitted amplitude is measured at a single point in space and time, at the detector. The spatial phases of the incident beam are modified to optimize this amplitude. The result is a short transmitted pulse at the target location and time. Figure b reproduced from ref. 105 , (c) 2003 APS. Figure c adapted from ref. 65, (c) 2011 APS.

\section{Controlling the time and frequency degrees of freedom}

The presence of many spatial channels in the optical domain provides great flexibility for wavefront-shaping experiments. However, wavefront shaping only works for a narrow frequency range. By optimizing the focus intensity and then detuning the laser, van Beijnum and co-workers ${ }^{56}$ have shown that the effect of optimization is lost after the laser has been detuned by the speckle correlation frequency $\delta \omega$. Rather than being a limitation, this allows for even more degrees of freedom to control the waves ${ }^{36}$. An incident light pulse excites all the modes that exist within its bandwidth (Fig. 3a). A source emits light with many frequency components, each of which causes a different speckle pattern. Similarly, tuning the phase of multiple spatial components makes it possible to control the relative phases and amplitudes of the frequency components, thereby allowing the transmitted speckles to interfere constructively at a chosen time. This extra dimension of control drastically increases the possibilities offered to an experimentalist.

An experimentalist can select a point in space and measure the phase and amplitude of each uncorrelated frequency comprising the spectrum of the field. A very basic way of controlling this transient wave field involves adding all the uncorrelated speckles in phase on the chosen point at a specific time. This control can be achieved by conjugating the phase of each component of the measured polychromatic wave field and sending it again through the 
medium. Owing to reciprocity, and because all the speckles have been phase-matched to interfere constructively at the desired location, a spatiotemporally focused wave emerges from the complex medium. This approach is exactly the definition of a time-reversal experiment because time reversing a signal in the temporal domain amounts to conjugating the phase of each frequency component in its spectrum.

A typical time-reversal experiment employs an array of transducers that are able to record the temporal modulation of the wave field and emit any temporal waveform. First, one records and digitizes the impulse response (the time-dependent Green's function) between a desired focal position and an array of sensors referred to as the time-reversal mirror (TRM). This impulse response is time reversed (chronologically inversed through the $t \rightarrow-t$ transform) and sent back from the array of sensors. This experiment was first realized in acoustics by Fink and co-workers ${ }^{29,57,58}$ using ultrasonic waves, piezoelectric transducers and a multiply scattering medium made out of randomly positioned steel rods immersed in a water tank (Fig. 3b). The researchers used a transducer to send a $1 \mu$ s pulse with a central frequency of $1.5 \mathrm{MHz}$ through the forest of steel rods and used a set of transducers to measure the field on the other side of the medium. The signal, which lasted for around 100 times the initial duration of the emitted pulse, was then time reversed and sent back from the transducer, and the field created by time reversal was measured in a volume around the original source position using a transducer on a translation stage. This resulted in an ultrasonic wave that was tightly focused in space and time - a few microseconds long and a few wavelengths wide - surrounded by a residual ultrasonic field known as 'spatiotemporal sidelobes' of average energy $N$ times lower than the peak ${ }^{59,60}$. Researchers also demonstrated time reversal in the electromagnetic domain using modulators that conjugate only the signal bandwidth rather than the full carrier bandwidth ${ }^{61,62}$.

Transferring the concepts of time reversal and related temporal methods ${ }^{63,64}$ to the optical domain is a challenge because it requires an interferometric measurement of the impulse responses, as well as a means of shaping the light pulses in phase and amplitude. This is now becoming possible thanks to recent advances on the spatiotemporal control of optical wave fields through random media ${ }^{65-67}$. Two of these demonstrations ${ }^{65,66}$ relied on the optimization a pulse's peak intensity after it had propagated through a complex medium, either by heterodyne interferometry (Fig. 3c) or by using a two-photon fluorescent material. These results were an important development over the earlier work of Aeschlimann and colleagues ${ }^{68}$, who focused light onto plasmonic nanoparticles by employing a feedback-based optimization technique to polarization-shape a light pulse. In the third demonstration ${ }^{67}$, McCabe and colleagues directly measured the complex spectrum of the optical field at a point in space and then phase-shaped the light pulse such that it focused in space and time at the chosen location.

There have been several theoretical proposals for achieving time reversal at optical frequencies. Yariv ${ }^{69}$ and Miller $^{70}$ independently proposed the use of four-wave mixing to compensate for the dispersion of a spread optical pulse. Yanik and Fan then numerically demonstrated the possibility of time-reversing optical pulses by dynamically modulating the refractive index of a dielectric photonic crystall $^{71}$, and Longhi later expanded this concept to the use of Bloch oscillations $^{72}$. Sivan and Pendry suggested using the dynamic modulation of a zero-gap periodic system to time-reverse a broadband light pulse $\mathrm{e}^{73}$, and Pendry established the link between time reversal and negative refraction ${ }^{74}$, which was subsequently verified experimentally by Katko and co-workers ${ }^{75}$.

\section{The medium as the lens}

Scanning a laser focus in a plane or volume allows an image to be constructed. Vellekoop and Aegerter ${ }^{76}$ have demonstrated this by combining wavefront shaping with a remarkable speckle correlation known as the memory effect ${ }^{77,78}$. Rotating the incident field by a small angle also rotates the transmitted field. This effect is shown in Fig. 4c. The incident field here consists of an array of spots, and tilting the incident field corresponds to phase-shifting the spots relative to each other. The transmitted spots are phase-shifted in the same way; that is, the transmitted field is rotated by the same angle. Using this rotation, a focus obtained by a one-time wavefront optimization can be scanned through the object plane to form an image. However, the correlation angle, which defines the field of view, is only appreciable for thin films of (at most) tens of wavelengths in thickness.

Hsieh et al. established an important generalization of this principle $^{79}$. They used digital phase conjugation instead of wavefront shaping to make the initial focus, and positioned a nanoparticle with a nonlinear response behind a scattering medium. When excited with a strong infrared laser pulse, the nanoparticle acted as a source of frequency-doubled blue light. The blue light propagated through a scattering medium towards the digital phase conjugation apparatus. The phase-conjugated blue light was focused back on the nanoparticle. By adding a phase gradient to the phase conjugated light, the researchers were able to scan the focus in the plane of the nanoparticle and thus image its surroundings.

In medical imaging, it is often undesirable to place a detector or a source inside a medium. A method for focusing light at a chosen position in a turbid medium would therefore be of great value. An important step in this direction was made by $\mathrm{Xu}$ and co-workers ${ }^{80}$, who employed an acousto-optic technique. They illuminated a sample with light and ultrasound simultaneously. The ultrasound waves acted as a source of frequency-shifted light waves, which the researchers separated from the unshifted light, phase-conjugated and then sent back to the focus. This method allows the light focus to be scanned at will because it tracks the ultrasound focus.

So far, this Review has not discussed the spatial characteristics of the focus in complex media - an aspect of major importance that has led to some surprising results. The first demonstration of focusing in opaque media took place with ultrasonic waves in water containing an array of steel rods $s^{58,60}$ (Fig. 3b). The researchers in this work used an array of transducers as a TRM to focus through the water-steel scattering medium onto distant points. They measured the transverse size of the focus as well as the focus obtained through water without steel rods. The results were surprising: the focus obtained through the scattering sample, although having an amplitude similar to that measured in water, had dimensions that were an order of magnitude lower.

This super-focusing technique is explained in Fig. 4a. In a homogeneous medium, a spatially extended source comprising an array of point sources, which are controlled in phase and amplitude to focus on a distant point, creates a diffraction pattern whose width $W=\lambda F / D$, where $D$ is the source aperture, $\lambda$ is the wavelength and $F$ is the focal distance. When the scattering medium is inserted between the source and the focus, the waves emitted from the source diffuse inside the multiply scattering medium. The diffusive halo acts as an array of Huygens secondary sources at the exit of the complex medium, along the interface that faces the focus. Because all these secondary sources are phase-matched by time reversal (or phase conjugation) to focus at the desired location, this array is spread across the entire medium and thus has a corresponding diffraction limit that is smaller than that of the aperture-limited lens (Fig. 4a). In this way, TRMs and wavefront-shaping apparatus use complex environments to appear wider than they are, resulting in a refocusing quality that no longer depends on their own angular aperture but rather on the angular aperture of the scattering medium, which can be up to $4 \pi$ steradians.

Such an increase in numerical aperture was first observed in acoustics using time reversal ${ }^{58}$, and later in microwaves through 

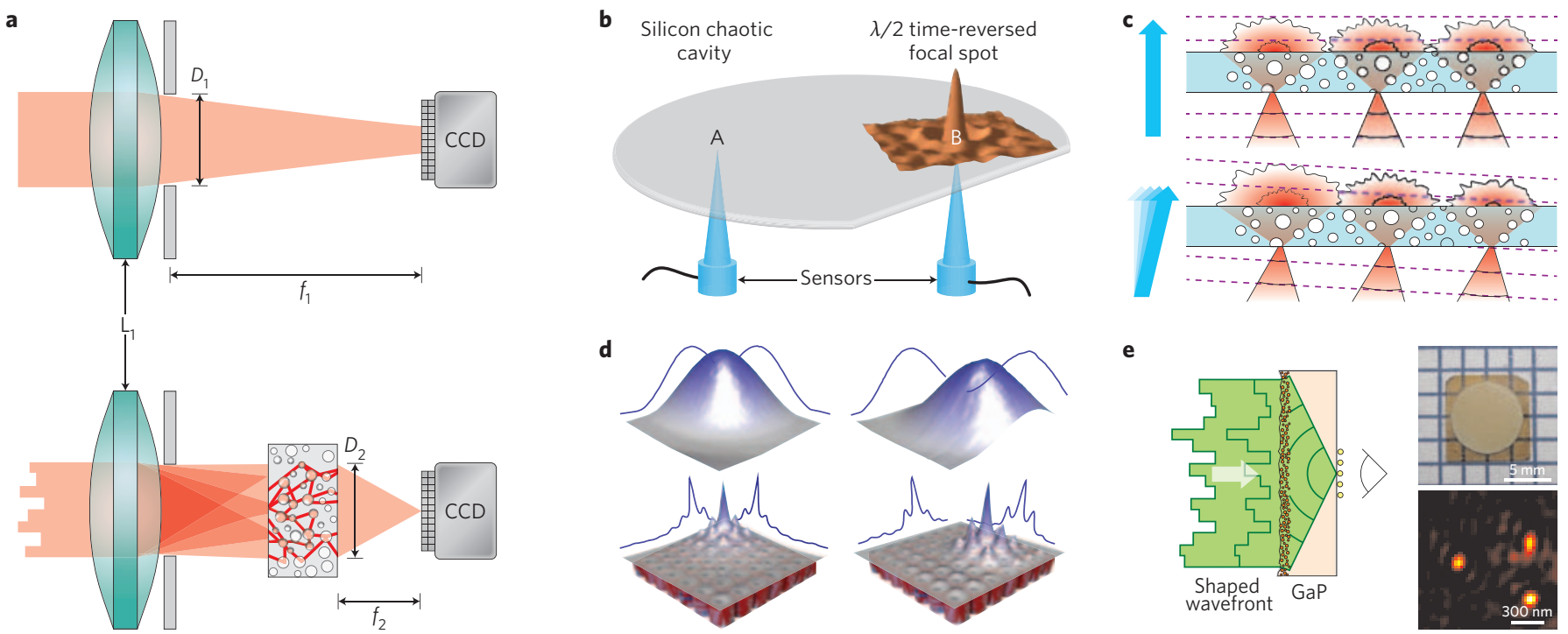

e
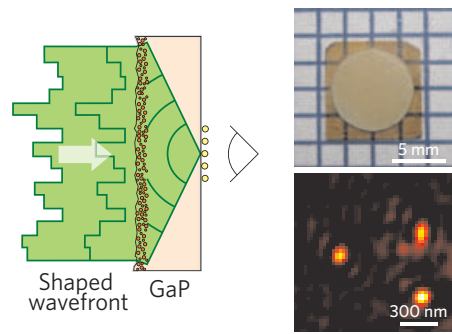

Figure 4 | Controlling waves in complex media for sub-diffraction and subwavelength focusing and imaging. a, Focusing a laser through a lens results in a focus that is limited by the numerical aperture of the lens, $\lambda f_{1} / D_{1}$, where $f_{1}$ is the focal length of the lens and $D_{1}$ is the diameter of the focus. Wavefront shaping through a complex medium creates a smaller focus because of the higher numerical aperture of the medium, $\lambda f_{2} / D_{2}$. $\mathbf{b}$, Time reversal of elastic waves in a silicon cavity. A single source at $A$ emits the time-reversed impulse response from $A$ to $B$, hence leading to a $\lambda / 2$-wide measured focal spot around B. c, The memory effect for waves. Adding a small phase gradient to the incident wave (here represented by an array of foci) will result in the same

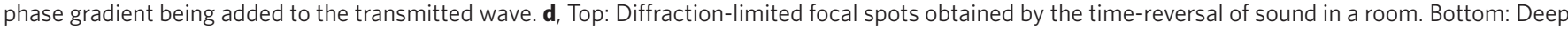
subwavelength focal spots obtained in an array of Helmholtz resonators using a method similar to time reversal. e, A wavefront-shaped beam optimized to focus at the interface of a high-refractive-index medium (gallium phosphide, top right). Scanning the focus using the memory effect yields a highresolution image of gold spheres (bottom right). Figure reproduced from: a, ref. 83, (c) 2010 NPG; b, ref. 85, (c) 1997 APS; d, ref. 91 , (C) 2011 APS; e, ref. 96 , (c) 2011 APS. Figure c provided by E. G. van Putten.

time reversal and phase-conjugation experiments ${ }^{62,81}$. In optics, a thin layer of white paint can be used to focus a laser spot as tightly as a very high numerical aperture converging lens. The layer of paint - a multiply scattering medium — is placed between an SLM and a CCD (Fig. 4a). After shaping the wavefront, researchers found the focus to be ten times tighter than that obtained without scattering ${ }^{82,83}$. In phase-conjugation experiments, researchers also observed that scattering in biological tissue can lead to an increase in image resolution ${ }^{84}$.

For broadband signals, the effect of increasing the numerical aperture is even more striking because the time-reversal operation only needs to be made from a single source, and the size of the focus depends only on the numerical aperture of the propagation medium, viewed from the focal point. This was demonstrated in an ultrasound experiment where a broadband signal was generated in a silicon wafer, which acted as reverberant cavity. The signal was time-reversed at a single point-like transducer, and using the cavity itself as a 'virtual lens' resulted in a focal spot as small as the diffraction limit ${ }^{85}$ (Fig. 4b).

Waves can be controlled inside multiply scattering media, similar to how they can be focused by scattering from the walls of a cavity. In such a situation the medium itself also behaves like a lens because it provides focusing down to a spot size that is no longer limited by the numerical aperture of the source, but rather by the scattering mean free path of the medium. Such an extreme increase in numerical aperture was demonstrated by van Putten and colleagues, who optimally focused light in a turbid medium ${ }^{86}$ and measured the corresponding focus using nanosized fluorescent spheres. Another simple and cost-effective $4 \pi$ microscopy scheme was proposed by Mudry and colleagues, who replaced the delicate two objectives with a combination of wavefront shaping, a single microscope objective and a mirror ${ }^{87}$.

The resolution-enhancement techniques mentioned so far involve increasing the numerical aperture of the system; they all involve exploiting a medium's complexity to focus to spots much smaller than those that can be achieved using a given source. In the aforementioned cases, however, the focus is still limited by the medium's index of refraction. In 2007, Lerosey and colleagues pursued a different approach by exploiting the possibilities offered by time reversal in a subwavelength-structured, multiply scattering medium. They showed that microwaves can be focused using time reversal from the far field inside a strongly scattering random collection of closely spaced metallic wires, down to foci as small as $\lambda / 30$, with very good efficiency ${ }^{88}$. Li and Stockman proposed the use of a randomly structured thin film of noble metal to create subwavelength hot spots from the far field using time reversal ${ }^{89}$. This led to the proposal of a resonant metalens - a lens capable of achieving subwavelength resolution in the far field - for imaging and focusing applications. This concept, which is based on coupled subwavelength resonators, uses polychromatic sources to take advantage of the spatiotemporal degrees of freedom offered by arrays of subwavelength resonant scatterers ${ }^{90}$. Using this approach, scientists also showed that sound can be controlled in the far field and focused to spots as small as $\lambda / 25$ (ref. 91) using an array of soda cans as Helmholtz resonators (Fig. 4d). Numerical simulations based on this idea ${ }^{92}$ have shown that wavefront shaping can be used to focus monochromatic light to subwavelength spots in an array of split rings. In this monochromatic approach, coupling between resonators gives rise to spatial sidelobes that are suppressed when using polychromatic signals ${ }^{93,94}$.

Wavefront shaping was recently extended into the realm of plasmonics by Gjonaj and co-workers, who focused plasmons using metal hole arrays in the far field, which is expected to bring the subwavelength resolution associated with these waves within reach soon $^{95}$. In another approach, researchers created a highly scattering interface on a high-refractive-index gallium phosphide medium (Fig. 4e) and used wavefront shaping to focus light close to the strongly reduced diffraction limit of this medium. They scanned the 

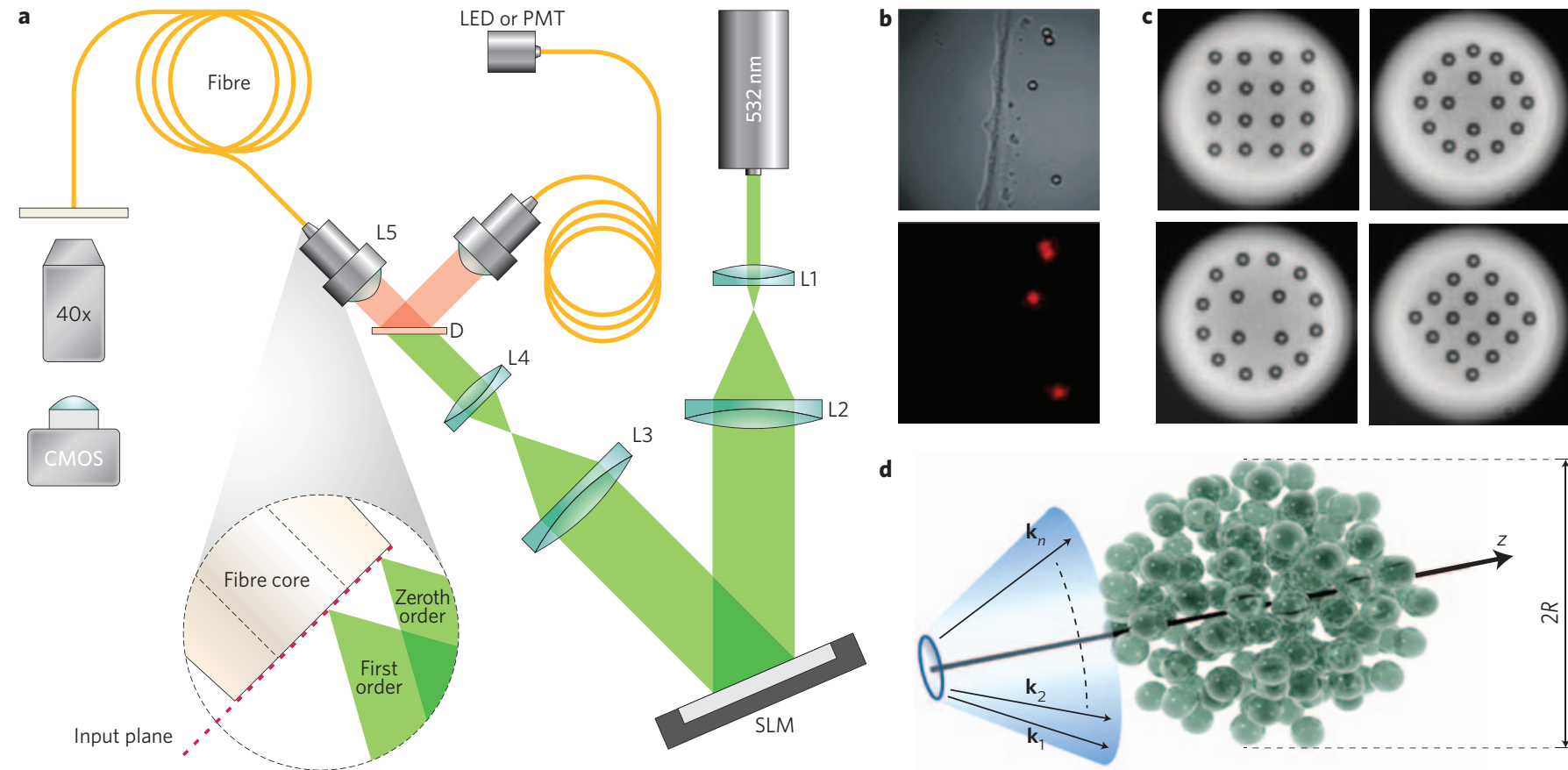

d
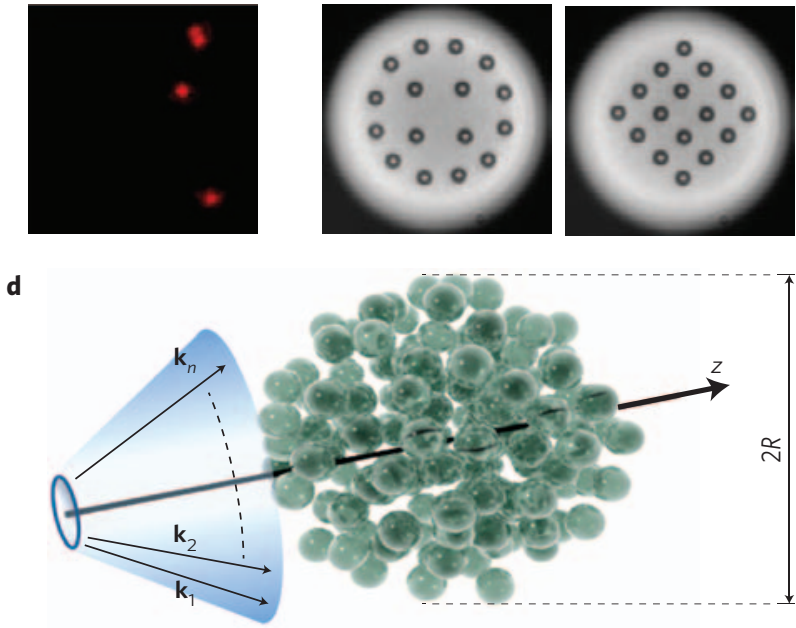

Figure 5 | Recent applications of wavefront control in complex media. a, An SLM can be used to measure the transmission matrix of a multimode fibre. Using this propagator in combination with wavefront shaping makes it possible to transmit microholograms through a 1-m-long, 60- $\mu$ m-diameter-core multimode fibre. This can be used to create spots across the output for performing scanning fluorescence microscopy. A dichroic mirror (D) allows the intensity to be measured with a photomultiplier tube (PMT). L1-L4, telescope lens; L5, fibre coupling lens. b, Bright-field image of $2 \mu \mathrm{m}$ fluorescent beads (top), and the same beads as seen by the transmission matrix approach based on a fibre fluorescence microscope (bottom). c, Optimizationbased wavefront shaping provides a tool for the micromanipulation of objects through multimode fibres. This image shows the two-dimensional optical manipulation of 16 polystyrene microparticles, each $3 \mu \mathrm{m}$ in diameter. The trapping beams (output modes) and the white-light illumination are both delivered by the multimode optical fibre. $\mathbf{d}$, A wavefront-shaped optical 'tractor beam' can exert pulling forces ( $\mathbf{k}$ ) on a collection of scatterers with radius R. Figure reproduced from: a,b, ref. 113, (c) 2012 RSC; c, ref. 114, (c) 2011 OSA; d, ref. 123, (c) 2011 APS.

focus using the memory effect, which enabled imaging at a resolution of $100 \mathrm{~nm}$ with green light ${ }^{96}$. Researchers have also proposed the use of wavefront shaping with a variety of nanostructures, including metal-dielectric multilayers ${ }^{97}$, gratings ${ }^{98}$ and coupled nano-particles ${ }^{99}$, in order to focus light from the far field onto spots as small as $\lambda / 30$.

\section{Applications and perspective}

The spatiotemporal control of waves in complex media offers exciting possibilities for many applications. Important capabilities in the ultrasound and radiofrequency domains have been reported, and some of these have already been applied to lightwave frequencies. Several new applications in optical imaging are emerging, and the fruitful interaction between the fields of optics and acoustics is giving rise to many applications that are still speculative.

In acoustics, time reversal has successfully been employed for the selective destruction of biological tissue by depositing high-power pulses using reverberant cavities ${ }^{100}$. The same principle was used to generate intense electromagnetic pulses for electronic warfare ${ }^{101}$. Ultrasound imaging in two and even three dimensions was demonstrated with only a few stationary sensors using a multiply scattering medium or a reverberant cavity, making use of the temporal degrees of freedom ${ }^{102}$. Many imaging schemes in random media have been proposed based on time reversal ${ }^{103}$. This is a major topic of interest in wireless communications - both in electromagnetism and in underwater acoustics - because it compensates for the distortions induced by a complex communication channel, maximizes the energy transfer, spatially multiplexes information and provides a means of physically coding information ${ }^{81,104-106}$. Indeed, as was shown by Moustakas and colleagues ${ }^{107}$, the information capacity of a medium can be significantly improved by scattering. Researchers recently proposed the use of time reversal for developing new sensors, by applying the quantum concept of fidelity to classical waves ${ }^{108}$.

In the optical domain, spatial wavefront shaping has been a key element in many new methods that aim to harness complex media. Aside from its applications in imaging, spatial wavefront shaping can be used to form optical tweezers in strongly scattering media ${ }^{109}$ and enhance the sensitivity of spectroscopy ${ }^{110}$. Furthermore, experimental confirmation of the existence of open transmission channels ${ }^{44}$ and the ability to measure a complex medium's transmission matrix show that wavefront control is useful for studying the fundamental physics of wave propagation ${ }^{41,44,45,47}$. Wavefront shaping has also recently been proposed as way of transmitting information through complex media ${ }^{50}$ to selectively focus light on nano-objects ${ }^{111}$, or for the Bessel beam light-sheet microscopy of scattering samples ${ }^{112}$.

Combining optical fibres with the ideas of wavefront shaping and transmission matrix measurements is incredibly powerful. Bianchi and Di Leonardo ${ }^{113}$ have demonstrated that a multimode fibre can be turned into a microscope by employing these techniques. In their set-up (Fig. 5a), light is injected in the multimode fibre after being phase-modulated by an SLM. The transmitted light is observed on a camera and an iterative algorithm makes it possible to transmit arbitrary intensity patterns. The performance of this set-up as a microscope is shown in Fig. 5b. Closely related to this study is the work of Cižmár and Dholakia (Fig. 5c), who achieved optical manipulation using a multimode fibre $\mathrm{e}^{114}$.

A significant limitation of early wavefront-control experiments was their low operating speed. To enable wavefront control in living tissue, optimizations must be done on a millisecond timescale. 
Phase-conjugation experiments in vivo have provided a weak signal that persists for less than a second ${ }^{115}$. Several groups have recently demonstrated wavefront shaping and transmission matrix measurements ${ }^{116-118}$ at optimization speeds 100 times faster than first-generation experiments ${ }^{39}$. This advance could allow deep imaging using two-photon excitation. Frequency-domain control of dispersion can already be used to improve multiphoton excitation in tissue ${ }^{119}$.

An imminent goal in optics is the ability to generate subwavelength-sized foci, following successful demonstrations in the fields of microwaves and acoustics ${ }^{88,91}$. Wavefront control methods and precisely nanostructured media or metamaterials could allow light to be focused at the $50 \mathrm{~nm}$ scale $e^{90,92,95,120}$, thereby opening new avenues of study in light-matter interactions, single-molecule sensing, nanolithography and nanoscale imaging.

Another prospect is an optical TRM that can control both the spatial and frequency degrees of freedom of light. Current experiments ${ }^{65-67}$ control either the frequency domain or the spatial domain, but not both. An optical TRM will open up an entirely new class of time-reversed optics experiments, yielding new fundamental insights into wave-scattering phenomena ${ }^{22-24,27,121}$. Important applications of TRMs are expected in the field of ultrafast nano-optics ${ }^{68,120,122}$

Several remarkable theoretical proposals that rely on wavefront control have recently been put forward. Sukhov and Dogariu proposed a wavefront-shaped optical 'tractor beam' that could exert a pulling force on scattering objects (Fig. 5d) ${ }^{123}$. Chong and Stone have theoretically demonstrated the existence of wavefronts that can be fully absorbed in a scattering medium, which may be of use in the conversion of light energy to heat or electricity ${ }^{124}$. Control of the incident wavefront may also be essential in achieving nanoscale lithography ${ }^{125}$.

A major driving force in the control of light in complex media has been the availability of sensitive and fast megapixel digital sensors and modulators. As in the ultrasound domain, programmable digital equipment will soon replace many of today's analog modulators and phase plates. A number of recent studies have used interference in the digital control of scattered light, which demonstrates that these methods are sufficiently well-developed for integration into applications. Through combinations with nonlinear optics, ultrasound or metamaterial approaches, and thanks to the participation of researchers from the many facets of optical science, the full power of wave control in complex media is now being realized.

\section{References}

1. Ishimaru, A. Wave Propagation and Scattering in Random Media (Academic, 1978).

2. Sebbah, P. Waves and Imaging through Complex Media (Kluwer Academic, 1999).

3. Tuchin, V. Tissue Optics: Light Scattering Methods and Instruments for Medical Diagnosis (SPIE, 2007)

4. Gibson, A. P., Hebden, J. C. \& Arridge, S. R. Recent advances in diffuse optical imaging. Phys. Med. Biol. 50, R1-R43 (2005)

5. Kennedy, L. C. et al. A new era for cancer treatment: Gold-nanoparticle-mediated thermal therapies. Small 7, 169-183 (2011).

6. Mady, E., Nadejda, M. \& Pascal, C. Review of several optical non-destructive analyses of an easel painting: Complementarity and crosschecking of the results. J. Cult. Herit. 12, 335-345 (2011).

7. Koenderink, A. F., Lagendijk, A. \& Vos, W. L. Optical extinction due to intrinsic structural variations of photonic crystals. Phys. Rev. B 72, $153102(2005)$

8. Leith, E. N. \& Upatnieks, J. Holographic imagery through diffusing media. J. Opt. Soc. Am. 56, 523-523 (1966).

9. Freund, I. Looking through walls and around corners. Physica A 168, 49-65 (1990).

10. Goodman, J. W. Statistical Optics (Wiley, 2000)

11. Sheng, P. Introduction to Wave Scattering, Localization and Mesoscopic Phenomena (Academic, 1995).

12. Beenakker, C. W. J. Random-matrix theory of quantum transport. Rev. Mod. Phys. 69, 731-808 (1997).

13. Andrews, L., Phillips, R. \& Hopen, C. Laser Beam Scintillation with Applications Vol. 99 (SPIE, 2001).
14. Huang, D. et al. Optical coherence tomography. Science 254, 1178-1181 (1991).

15. Helmchen, F. \& Denk, W. Deep tissue two-photon microscopy. Nature Meth. 2, 932-940 (2005).

16. den Outer, P. N., Nieuwenhuizen, T. \& Lagendijk, A. Location of objects in multiple-scattering media. J. Opt. Soc. Am. A 10, 1209-1218 (1993).

17. Ntziachristos, V. Fluorescence molecular imaging. Ann. Rev. Biomed. Eng. 8, 1-33 (2006).

18. Bridges, W. B. et al. Coherent optical adaptive techniques. Appl. Opt. 13, 291-300 (1974).

19. Hardy, J. W. Adaptive Optics for Astronomical Telescopes (Oxford Univ., 1998)

20. Dainty, J. C. in Laser Speckle and Related Phenomena Vol. 9, 255-280 (Springer, 1975).

21. Chandrasekhar, S. Radiative Transfer (Dover, 1960).

22. van Albada, M. P. \& Lagendijk, A. Observation of weak localization of light in a random medium. Phys. Rev. Lett. 55, 2692-2695 (1985).

23. Wolf, P. E. \& Maret, G. Weak localization and coherent backscattering of photons in disordered media. Phys. Rev. Lett. 55, 2696-2699 (1985).

24. Wiersma, D. S., Bartolini, P., Lagendijk, A. \& Righini, R. Localization of light in a disordered medium. Nature 390, 671-673 (1997).

25. Chabanov, A. A., Stoytchev, M. \& Genack, A. Z. Statistical signatures of photon localization. Nature 404, 6780 (2000).

26. Hu, H., Strybulevych, A., Page, J., Skipetrov, S. \& Van Tiggelen, B. Localization of ultrasound in a three-dimensional elastic network. Nature Phys. 4, 945-948 (2008).

27. Lagendijk, A., van Tiggelen, B. \& Wiersma, D. S. Fifty years of Anderson localization. Phys. Today 62, 24-29 (August 2009).

28. Kop, R. H. J., Vries, P. D., Sprik, R. \& Lagendijk, A. Observation of anomalous transport of strongly multiple scattered light in thin disordered slabs. Phys. Rev. Lett. 79, 4369-4372 (1997).

29. Fink, M. Time reversed acoustics. Phys. Today 50, 34-40 (March 1997).

30. van Albada, M. P., de Boer, J. F. \& Lagendijk, A. Observation of long-range intensity correlation in the transport of coherent light through a random medium. Phys. Rev. Lett. 64, 2787-2790 (1990).

31. van Albada, M. P., van Tiggelen, B. A., Lagendijk, A. \& Tip, A. Speed of propagation of classical waves in strongly scattering media. Phys. Rev. Lett. 66, 3132-3135 (1991)

32. Lagendijk, A. \& van Tiggelen, B. A. Resonant multiple scattering of light. Phys. Rep. 270, 143-215 (1996).

33. Wang, J. \& Genack, A. Transport through modes in random media. Nature 471, 345-348 (2011).

34. Boer, J. F. D., Albada, M. P. V. \& Lagendijk, A. Transmission and intensity correlations in wave-propagation through random-media. Phys. Rev. B 45, 658-666 (1992).

35. Emiliani, V. et al. Near-field short range correlation in optical waves transmitted through random media. Phys. Rev. Lett. 90, 250801 (2003).

36. Lemoult, F., Lerosey, G., de Rosny, J. \& Fink, M. Manipulating spatiotemporal degrees of freedom of waves in random media. Phys. Rev. Lett. 103, 173902 (2009).

37. Davy, M., Shi, Z. \& Genack, A. Z. Focusing through random media: Eigenchannel participation number and intensity correlation. Phys. Rev. B $\mathbf{8 5}$, 035105 (2012).

38. Maurer, C., Jesacher, A., Bernet, S. \& Ritsch-Marte, M. What spatial light modulators can do for optical microscopy. Laser Photon. Rev. 5, 81-101 (2011).

39. Vellekoop, I. M. \& Mosk, A. P. Focusing coherent light through opaque strongly scattering media. Opt. Lett. 32, 2309-2311 (2007)

40. Vellekoop, I. M., van Putten, E. G., Lagendijk, A. \& Mosk, A. P. Demixing light paths inside disordered metamaterials. Opt. Express 16, 67-80 (2008).

41. Pendry, J. B. Light finds a way through the maze. Physics 1, 20 (2008).

42. Dorokhov, O. N. On the coexistence of localized and extended electronic states in the metallic phase. Sol. Stat. Commun. 51, 381-384 (1984).

43. Imry, Y. Active transmission channels and universal conductance fluctuations. Europhys. Lett. 1, 249-256 (1986).

44. Vellekoop, I. M. \& Mosk, A. P. Universal optimal transmission of light through disordered materials. Phys. Rev. Lett. 101, 120601 (2008).

45. Choi, W., Mosk, A. P., Park, Q.-H. \& Choi, W. Transmission eigenchannels in a disordered medium. Phys. Rev. B 83, 134207 (2011).

46. Shi, Z. \& Genack, A. Z. Transmission eigenvalues and the bare conductance in the crossover to Anderson localization. Phys. Rev. Lett. 108, 043901 (2012).

47. Popoff, S. et al. Measuring the transmission matrix in optics: An approach to the study and control of light propagation in disordered media. Phys. Rev. Lett. 104, 100601 (2010).

48. van Putten, E. G. \& Mosk, A. P. The information age in optics: Measuring the transmission matrix. Physics 3, 22 (2010).

49. Kohlgraf-Owens, T. \& Dogariu, A. Transmission matrices of random media: Means for spectral polarimetric measurements. Opt. Lett. 35, $2236-2238$ (2010). 
50. Popoff, S., Lerosey, G., Fink, M., Boccara, A. \& Gigan, S. Image transmission through an opaque material. Nature Commun. 1, 1-5 (2010).

51. Yariv, A. \& Pepper, D. Amplified reflection, phase conjugation, and oscillation in degenerate four-wave mixing. Opt. Lett. 1, 16-18 (1977).

52. Zel'dovich, B., Popovichev, V., Ragul'skii, V. \& Faizullov, F. Connection between the wave fronts of the reflected and exciting light in stimulated Mandel'shtam-Brillouin scattering. JETP Lett. 15, 109-112 (1972).

53. Günter, P. \& Huignard, J. Photorefractive Materials and their Applications I: Materials Vol. 2 (Springer, 2007).

54. Yaqoob, Z., Psaltis, D., Feld, M. S. \& Yang, C. Optical phase conjugation for turbidity suppression in biological samples. Nature Photon. 2, 110-115 (2008).

55. Cui, M. \& Yang, C. Implementation of a digital optical phase conjugation system and its application to study the robustness of turbidity suppression by phase conjugation. Opt. Express 18, 3444-3455 (2010).

56. Van Beijnum, F., van Putten, E., Lagendijk, A. \& Mosk, A. P. Frequency bandwidth of light focused through turbid media. Opt. Lett. 36, 373-375 (2011).

57. Fink, M. Time reversal of ultrasonic fields. I: Basic principles. IEEE Trans. Ultrason. Ferroelectr. Freq. Control 39, 555-566 (1992).

58. Derode, A., Roux, P. \& Fink, M. Robust acoustic time reversal with highorder multiple scattering. Phys. Rev. Lett. 75, 4206-4209 (1995).

59. Derode, A., Tourin, A. \& Fink, M. Random multiple scattering of ultrasound. I: Coherent and ballistic waves. Phys. Rev. E 64, 036605 (2001).

60. Derode, A., Tourin, A. \& Fink, M. Random multiple scattering of ultrasound. II: Is time reversal a self-averaging process? Phys. Rev. E 64, 036606 (2001).

61. Lerosey, G. et al. Time reversal of electromagnetic waves. Phys. Rev. Lett. 92, 193904 (2004).

62. Lerosey, G., de Rosny, J., Tourin, A., Derode, A. \& Fink, M. Time reversal of wideband microwaves. Appl. Phys. Lett. 88, 154101 (2006).

63. Tanter, M., Aubry, J. F., Gerber, J., Thomas, J. L. \& Fink, M. Optimal focusing by spatio-temporal inverse filter. I: Basic principles. J. Acoust. Soc. Am. 110, 37-47 (2001).

64. Montaldo, G., Tanter, M. \& Fink, M. Real time inverse filter focusing through iterative time reversal. J. Acoust. Soc. Am. 115, 768-775 (2004).

65. Aulbach, J., Gjonaj, B., Johnson, P. M., Mosk, A. P. \& Lagendijk, A. Control of light transmission through opaque scattering media in space and time. Phys. Rev. Lett. 106, 103901 (2011).

66. Katz, O., Small, E., Bromberg, Y. \& Silberberg, Y. Focusing and compression of ultrashort pulses through scattering media. Nature Photon. 5, 372-377 (2011).

67. McCabe, D. et al. Spatio-temporal focusing of an ultrafast pulse through a multiply scattering medium. Nature Commun. 2, 447 (2011)

68. Aeschlimann, M. et al. Adaptive subwavelength control of nano-optical fields. Nature 446, 301-304 (2007).

69. Yariv, A. Four wave nonlinear optical mixing as real time holography. Opt. Commun. 25, 23-25 (1978).

70. Miller, D. A. B. Time reversal of optical pulses by four-wave mixing. Opt. Lett. 5, 300-302 (1980)

71. Yanik, M. F. \& Fan, S. Time reversal of light with linear optics and modulators. Phys. Rev. Lett. 93, 173903 (2004).

72. Longhi, S. Stopping and time reversal of light in dynamic photonic structures via Bloch oscillations. Phys. Rev. E 75, 026606 (2007).

73. Sivan, Y. \& Pendry, J. B. Time reversal in dynamically tuned zero-gap periodic systems. Phys. Rev. Lett. 106, 193902 (2011).

74. Pendry, J. B. Time reversal and negative refraction. Science 322, 71-73 (2008).

75. Katko, A. R. et al. Phase conjugation and negative refraction using nonlinear active metamaterials. Phys. Rev. Lett. 105, 123905 (2010).

76. Vellekoop, I. M. \& Aegerter, C. M. Scattered light fluorescence microscopy: Imaging through turbid layers. Opt. Lett. 35, 1245-1247 (2010).

77. Feng, S., Kane, C., Lee, P. A. \& Stone, A. D. Correlations and fluctuations of coherent wave transmission through disordered media. Phys. Rev. Lett. 61, 834-837 (1988).

78. Freund, I., Rosenbluh, M. \& Feng, S. Memory effects in propagation of optical waves through disordered media. Phys. Rev. Lett. 61, 2328-2331 (1988).

79. Hsieh, C., Pu, Y., Grange, R. \& Psaltis, D. Digital phase conjugation of second harmonic radiation emitted by nanoparticles in turbid media. Opt. Express 18, 12283-12290 (2010).

80. Xu, X., Liu, H. \& Wang, L. Time-reversed ultrasonically encoded optical focusing into scattering media. Nature Photon. 5, 154-157 (2011).

81. Henty, B. E. \& Stancil, D. D. Multipath-enabled super-resolution for RF and microwave communication using phase-conjugate arrays. Phys. Rev. Lett. 93, 243904 (2004)

82. Vellekoop, I. M. Controlling the Propagation of Light in Disordered Scattering Media. PhD thesis, Univ. Twente (2008).
83. Vellekoop, I. M., Lagendijk, A. \& Mosk, A. P. Exploiting disorder for perfect focusing. Nature Photon. 4, 320-322 (2010)

84. Cui, M., McDowell, E. J. \& Yang, C. Observation of polarization-gate based reconstruction quality improvement during the process of turbidity suppression by optical phase conjugation. Appl. Phys. Lett. 95, 123702 (2009).

85. Draeger, C. \& Fink, M. One-channel time reversal of elastic waves in a chaotic 2D-silicon cavity. Phys. Rev. Lett. 79, 407-410 (1997).

86. van Putten, E. G., Lagendijk, A. \& Mosk, A. P. Optimal concentration of light in turbid materials. J. Opt. Soc. Am. B 28, 1200-1203 (2011).

87. Mudry, E., Le Moal, E., Ferrand, P., Chaumet, P. C. \& Sentenac, A. Isotropic diffraction-limited focusing using a single objective lens. Phys. Rev. Lett. 105, 203903 (2010)

88. Lerosey, G., de Rosny, J., Tourin, A. \& Fink, M. Focusing beyond the diffraction limit with far-field time reversal. Science 315, 1120-1122 (2007).

89. Li, X. \& Stockman, M. I. Highly efficient spatiotemporal coherent control in nanoplasmonics on a nanometer-femtosecond scale by time reversal. Phys. Rev. B 77, 195109 (2008).

90. Lemoult, F., Lerosey, G., de Rosny, J. \& Fink, M. Resonant metalenses for breaking the diffraction barrier. Phys. Rev. Lett. 104, 203901 (2010).

91. Lemoult, F., Fink, M. \& Lerosey, G. Acoustic resonators for far-field control of sound on a subwavelength scale. Phys. Rev. Lett. 107, 064301 (2011).

92. Kao, T. S., Jenkins, S. D., Ruostekoski, J. \& Zheludev, N. I. Coherent control of nanoscale light localization in metamaterial: Creating and positioning isolated subwavelength energy hot spots. Phys. Rev. Lett. 106, 085501 (2011)

93. Lemoult, F., Fink, M. \& Lerosey, G. Revisiting the wire medium: An ideal resonant metalens. Wave. Random Complex 21, 591-613 (2011).

94. Lemoult, F., Fink, M. \& Lerosey, G. Far-field sub-wavelength imaging and focusing using a wire medium based resonant metalens. Wave. Random Complex 21, 614-627 (2011).

95. Gjonaj, B. et al. Active spatial control of plasmonic fields. Nature Photon. $\mathbf{5}$ 360-363 (2011)

96. van Putten, E. G. et al. Scattering lens resolves sub-100 nm structures with visible light. Phys. Rev. Lett. 106, 193905 (2011).

97. Bartal, G., Lerosey, G. \& Zhang, X. Subwavelength dynamic focusing in plasmonic nanostructures using time reversal. Phys. Rev. B 79, 201103 (2009)

98. Sentenac, A. \& Chaumet, P. Subdiffraction light focusing on a grating substrate. Phys. Rev. Lett. 101, 013901 (2008).

99. Volpe, G., Molina-Terriza, G. \& Quidant, R. Deterministic subwavelength control of light confinement in nanostructures. Phys. Rev. Lett. 105 216802 (2010).

100. Montaldo, G., Roux, P., Derode, A., Negreira, C. \& Fink, M. Ultrasound shock wave generator with one-bit time reversal in a dispersive medium, application to lithotripsy. Appl. Phys. Lett. 80, 897-899 (2002).

101. Davy, M., de Rosny, J., Joly, J.-C. \& Fink, M. Focusing and amplification of electromagnetic waves by time reversal in an leaky reverberation chamber. C. R. Phys. 11, 37-43 (2010)

102. Montaldo, G., Palacio, D., Tanter, M. \& Fink, M. Time reversal kaleidoscope: A smart transducer for three-dimensional ultrasonic imaging. Appl. Phys. Lett. 84, 3879-3881 (2004).

103. Borcea, L., Papanicolaou, G., Tsogka, C. \& Berryman, J. Imaging and time reversal in random media. Inverse Probl. 18, 1247-1279 (2002).

104. Kuperman, W. \& Lynch, J. Shallow-water acoustics. Phys. Today 57, 55-61 (October 2004)

105. Derode, A. et al. Taking advantage of multiple scattering to communicate with time reversal antennas. Phys. Rev. Lett. 90, 014301 (2003).

106. Lerosey, G. et al. Time reversal of electromagnetic waves and telecommunication. Radio Sci. 40, RS6S12 (2005).

107. Moustakas, A. L., Baranger, H. U., Balents, L., Sengupta, A. M. \& Simon, S. H. Communication through a diffusive medium: Coherence and capacity. Science 287, 287-290 (2000).

108. Taddese, B. T., Hart, J., Antonsen, T. M., Ott, E. \& Anlage, S. M. Sensor based on extending the concept of fidelity to classical waves. Appl. Phys. Lett. 95, 114103 (2009).

109. Cizmár, T., Mazilu, M. \& Dholakia, K. In situ wavefront correction and its application to micromanipulation. Nature Photon. 4, 388-394 (2010)

110. Svensson, T., Adolfsson, E., Lewander, M., Xu, C. T. \& Svanberg, S. Disordered, strongly scattering porous materials as miniature multipass gas cells. Phys. Rev. Lett. 107, 143901 (2011).

111. Popoff, S. M. et al. Exploiting the time reversal operator for adaptive optics, selective focusing, and scattering pattern analysis. Phys. Rev. Lett. 107, 263901 (2011).

112. Fahrbach, F., Simon, P. \& Rohrbach, A. Microscopy with self-reconstructing beams. Nature Photon. 4, 780-785 (2010).

113. Bianchi, S. \& Di Leonardo, R. A multi-mode fiber probe for holographic micromanipulation and microscopy. Lab Chip 12, 635-639 (2012). 
114. Cižmár, T. \& Dholakia, K. Shaping the light transmission through a multimode optical fibre: complex transformation analysis and applications in biophotonics. Opt. Express 19, 18871-18884 (2011).

115. Cui, M., McDowell, E. \& Yang, C. An in vivo study of turbidity suppression by optical phase conjugation (TSOPC) on rabbit ear. Opt. Express 18, 25-30 (2010).

116. Choi, Y. et al. Overcoming the diffraction limit using multiple light scattering in a highly disordered medium. Phys. Rev. Lett. 107, 023902 (2011).

117. Cui, M. A high speed wavefront determination method based on spatial frequency modulations for focusing light through random scattering media. Opt. Express 19, 2989-2995 (2011).

118. Conkey, D. B., Caravaca-Aguirre, A. M. \& Piestun, R. High-speed scattering medium characterization with application to focusing light through turbid media. Opt. Express 20, 1733-1740 (2012).

119. Dela Cruz, J., Pastirk, I., Comstock, M., Lozovoy, V. \& Dantus, M. Use of coherent control methods through scattering biological tissue to achieve functional imaging. Proc. Natl Acad. Sci. USA 101, 16996-17001 (2004).

120. Stockman, M. I. Ultrafast nanoplasmonics under coherent control. New J. Phys. 10, 025031 (2008).
121. Skipetrov, S. E. \& van Tiggelen, B. A. Dynamics of Anderson localization in open 3D media. Phys. Rev. Lett. 96, 043902 (2006).

122. Aeschlimann, M. et al. Spatiotemporal control of nanooptical excitations. Proc. Natl Acad. Sci. USA 107, 5329-5333 (2010).

123. Sukhov, S. \& Dogariu, A. Negative nonconservative forces: Optical 'tractor beams' for arbitrary objects. Phys. Rev. Lett. 107, 203602 (2011).

124. Chong, Y. D. \& Stone, A. D. Hidden black: Coherent enhancement of absorption in strongly scattering media. Phys. Rev. Lett. 107, 163901 (2011).

125. Srituravanich, W., Fang, N., Sun, C., Luo, Q. \& Zhang, X. Plasmonic nanolithography. Nano Lett. 4, 1085-1088 (2004).

\section{Acknowledgements}

A.P.M. acknowledges financial support from the European Research Council (grant number 279248). The authors thank E. G. van Putten for providing Fig. 4c.

\section{Additional information}

Correspondence and requests for materials should be addressed to A.P.M. 